\title{
Optimal Kernel-Based Dynamic Mode Decomposition
}

\section{ABSTRACT}

The state-of-the-art algorithm known as kernel-based dynamic mode decomposition (K-DMD) provides a sub-optimal solution to the problem of reduced modeling of a dynamical system based on a finite approximation of the Koopman operator. It relies on crude approximations and on restrictive assumptions. The
purpose of this work is to propose a kernel-based algorithm solving exactly this low-rank approximation problem in a general setting.

\section{CONTEXT AND PROBLEM}

- Intractable high-dimensional systems:

$$
\left\{\begin{array}{l}
x_{t}(\theta)=f_{t}\left(x_{t-1}(\theta)\right), \\
x_{1}(\theta)=\theta
\end{array}\right.
$$

where $x_{t} \in \mathbb{R}^{p}$ is the state variable, $f_{t}: \mathbb{R}^{p} \rightarrow \mathbb{R}^{p}$, and $\theta \in \Theta \subseteq \mathbb{R}^{p}$ denotes an initial condition

\section{- Idealised reduced model}

* Assume $\Psi \in\left(\mathcal{L}^{2}\left(\mathbb{R}^{p}\right)\right)^{n}$ such that its image is an invariant vector space of the Koopman operator.

*A finite approximation of the Koopman operator of rank $k \leq p$ approximates $x_{t}(\theta)$ by

$$
\tilde{x}_{t}(\theta)=\Psi^{-1}\left(\sum_{i=1}^{k} \lambda_{i}^{t-1} \xi_{i}^{\top} \Psi(\theta) \zeta_{i}\right)
$$

where $\left(\xi_{i}, \zeta_{i}, \lambda_{i}\right) \in \mathbb{C}^{n} \times \mathbb{C}^{n} \times \mathbb{C}$ target the left/right eigen-vectors and eigen-values of

$$
\underset{A \cdot \operatorname{rank}(A) \leq k}{\arg \min } \int_{\Theta} \sum_{t>1}\left\|\Psi\left(x_{t}(\theta)\right)-A^{t-1} \Psi\left(x_{1}(\theta)\right)\right\|_{2}^{2} d \theta .
$$

\section{- Data-driven reduced model:}

* Snapshots $\left\{x_{t}\left(\theta_{\ell}\right)\right\}_{t=1}^{T}, \ell=1, \ldots, N$, of $(1)$ for initial conditions $\theta_{\ell} \in \Theta$

$*$ Matrices $\Psi_{\mathbf{X}}=\left(\Psi_{1: T-1}^{1} \cdots \Psi_{1: T-1}^{N}\right)$ and $\Psi_{\mathbf{Y}}=\left(\Psi_{2: T}^{1} \cdots \Psi_{2: T}^{N}\right)$, in $\mathbb{R}^{n \times m}$ with $m=$ $N(T-1)$ and $\Psi_{t_{1}: t_{2}}^{\ell}=\left(\Psi\left(x_{t_{1}}\left(\theta_{\ell}\right)\right) \cdots \Psi\left(x_{t_{2}}\left(\theta_{\ell}\right)\right)\right)$.

$*$ Matrices $\mathbf{Y}=\Psi^{-1}\left(\Psi_{\mathbf{Y}}\right)$ and $\mathbf{X}=\Psi^{-1}\left(\Psi_{\mathbf{X}}\right)$ in $\mathbb{R}^{p \times m}$, where $\Psi$ is the identity operator.

Problem 1. Assume $A_{k}^{\star}$ is diagonalisable. Given the snapshots $x_{t}\left(\theta_{i}\right)$ 's and $\theta \in \Theta$, compute $\tilde{x}_{t}(\theta)$ solution of the reduced model $(2)$, where $\left(\xi_{i}, \zeta_{i}, \lambda_{i}\right)$ 's target the eigenvectors and eigen-values of

$$
A_{k}^{\star} \in \underset{A: \operatorname{rank}(A) \leq k}{\arg \min }\left\|\mathbf{\Psi}_{\mathbf{Y}}-A \mathbf{\Psi}_{\mathbf{X}}\right\|_{F}^{2} .
$$

\section{STATE-OF-THE-ART}

- In the low-rank DMD algorithm of [Heas 17$]$, exact computation of $\tilde{x}_{t}(\theta)$ is obtained from the eigendecomposition of the optimal solution

$$
A_{k}^{\star}=\mathcal{P P}^{\top} \Psi_{\mathbf{Y}} \Psi_{\mathrm{X}}^{\dagger},
$$

where columns of $\mathcal{P} \in \mathbb{R}^{n \times k}$ are the $k$-first left singular vectors of matrix $\Psi_{\mathbf{Y}} \mathbb{P}_{\Psi_{\mathbf{X}}^{\top}} \in \mathbb{R}^{n \times m}$.

$\Rightarrow$ complexity scales in $\mathcal{O}(n)$, which is prohibitive for large (possibly infinite) $n \gg p$.

- In [Williams14\}), the K-DMD algorithm approximates $\tilde{x}_{t}(\theta)$ using the "kernel trick" with a complexity in $\mathcal{O}(p)$, independent of $n . \Rightarrow$ sub-optimal solution of $\tilde{x}_{t}(\theta)$ because it assumes that: iv) low-rank constraint can be ignored

\section{UPGRADING K-DMD}

\section{- The "kernel trick"}

$\underset{\text { if there exists }}{*} \Psi_{\mathbf{Y}}^{\top} \Psi_{\mathbf{Y}}, \Psi_{\mathbf{Y}}^{\top} \Psi_{Y}, \Psi_{Y}^{\top} \Psi_{X}$ and $\Psi_{X}^{\top} \Psi(\theta)$ for any $\theta \in \Theta$ are computable from $X$ and $\mathbf{Y}$ in $\mathcal{O}(p)$ if there exists $\quad h: \mathbb{R}^{p} \times \mathbb{R}^{p} \rightarrow \mathbb{R} ; \quad(y, z) \rightarrow h(y, z)=\Psi(y)^{\top} \Psi(z)$.

\section{- Kernel-based representation of eigen-vectors:}

$*\left\{\left(\tilde{\xi}_{i}, \tilde{\lambda}_{\ell, i}\right\}_{i=1}^{k}\right.$ and $\left\{\left(\tilde{\zeta}_{i}, \tilde{\lambda}_{r, i}\right\}_{i=1}^{k}\right.$ are two sets of eigen-vectors/values of well-chosen matrices $\left(\tilde{A}_{\ell, k}\right)^{\top}$ and $\tilde{A}_{r, k}$ in $\mathbb{R}^{m \times m}$, computed in $\mathcal{O}(p)$ using the kernel trick.

* The following proposition exhibits a kernel-based low-dimensional representation of the eigendecomposition of $A_{k}^{\star}$ relying on the factorisation (3).

Proposition 1. For $i=1, \cdots, k$, the left and right eigen-vectors of $A_{k}^{\star}$ and its eigen-values satisfy $\xi_{i}=U_{\Psi_{X}} \tilde{\xi}_{i}$ and $\zeta_{i}=\mathcal{P} \tilde{\zeta}_{i}$ with $\quad \lambda_{i}=\tilde{\lambda}_{\ell, i}=\tilde{\lambda}_{r, i}$, where
columns of $U_{\Psi_{\mathbf{X}}}$ are the right eigen-vectors of $\Psi_{\mathbf{X}}$.

\section{• Kernel-based inversion:}

* From Proposition 1, we show that (2) is equivalent to $\tilde{x}_{t}(\theta)=\Psi^{-1}\left(\Psi_{\mathbf{Y}} G(\theta)\right)$,

$* G(\theta) \in \mathbb{R}^{m \times k}$ depends on $\tilde{\zeta}_{i}$ 's on $\lambda_{i}$ 's and $\tilde{\varphi}_{i}(\theta)$ 's, where the latter are eigen-functions derived in $\mathcal{O}(p)$ operations from $\tilde{\xi}_{i}$ 's and $\Psi_{\mathrm{X}}^{\mathrm{T}} \Psi(\theta)$.

* Computation of $\Psi^{-1}\left(\Psi_{\mathrm{Y}} G(\theta)\right)$ in $\mathcal{O}(p)$

1. Polynomial kernels.

$h(y, z)=\left(1+y^{\top} z\right)$ is a scalar product in $\left(\mathcal{L}^{2}\left(\mathbb{R}^{p}\right)\right)^{n}$ with $n<\infty$ $\cdot \Psi^{-1}\left(\Psi_{\mathbf{Y}} G(\theta)\right)=\mathbf{Y} G(\theta)$ (linear)

2. Gaussian kernels. $\cdot h(y, z)=\exp \left(-\frac{\|y-z\|_{2}^{2}}{2 \sigma^{2}}\right)$ is a scalar product in $\left(\mathcal{L}^{2}\left(\mathbb{R}^{p}\right)\right)^{n}$ with $n=\infty$

- $\Psi^{-1}\left(\Psi_{\mathbf{Y}} G(\theta)\right)=$ weighted sum of entries of $\mathbf{Y}$

3. Logarithmic kernel.

$h(y, z)=(\log (y+1))^{\top} \log (z+1)$ is a scalar product in $\left(\mathcal{L}^{2}\left(\mathbb{R}^{p}\right)^{n}\right.$ with $n=p$.

$\Psi^{-1}\left(\Psi_{\mathbf{Y}} G(\theta)\right)=$ product of exponentials of entries of $\mathbf{Y}$.
• Optimal K-DMD algorithm (OK-DMD)

inputs: The $x_{t}\left(\theta_{i}\right)$ 's and some $\theta$

1) Compute $\Psi_{\mathrm{X}}^{\top} \Psi_{\mathrm{X}}, \Psi_{\mathrm{Y}}^{\top} \Psi_{\mathrm{Y}}, \Psi_{\mathrm{Y}}^{\top} \Psi_{\mathrm{X}}$ and $\Psi_{\mathrm{X}}^{\top} \Psi(\theta)$ with the kernel trick

2) Derive matrices $\left(\tilde{A}_{\ell, k}\right)^{\top}$ and $\tilde{A}_{r, k}$.

3) Compute their eigen-vectors/values $\left\{\left(\tilde{\xi}_{i}, \tilde{\lambda}_{\ell, i}\right)\right\}_{i=1}^{k}$ and $\left\{\left(\tilde{\zeta}_{i}, \tilde{\lambda}_{r, i}\right)\right\}_{i=1}^{k}$.

4) Compute the eigen-functions $\left\{\tilde{\varphi}_{i}(\theta)\right\}_{i=1}^{k}$.

5) Derive $G(\theta)$ and compute $\tilde{x}_{t}(\theta)=\Psi^{-1}(\Psi(\mathbf{Y}) G(\theta))$ using the kernel-based inversion formulae. output: $x_{t}(\theta)$ 's

\section{NUMERICAL EVALUATION}

\section{- Toy model:}

* Snapshots of a balanced mixture of trajectories of a linear and a quadratic model.

$$
f\left(x_{t-1}\left(\theta_{\ell}\right)\right)=\left(x_{t-1}\left(\theta_{\ell}\right)+1\right)^{\beta}-1, \quad \beta \in\{1,2\},
$$

with an identical initial condition (i.i.d. realisations of the standard normal distribution).

* Normalised approximation error norm $\|\mathbf{Y}-\tilde{\mathbf{Y}}\|_{F} /\|\mathbf{Y}\|_{F}$, with the $i$-th column of $\tilde{\mathbf{Y}} \in \mathbb{R}^{p \times m}$ defined as $\tilde{x}_{2}(\theta)$ where $\theta$ is identified to the $i$-th column of $\mathbf{X}$

Remark: for low-rank DMD, this criterion simplifies into $\left\|\mathbf{Y}-A_{k}^{\star} \mathbf{X}\right\|_{F} /\|\mathbf{Y}\|_{F}$.

\section{Results:}
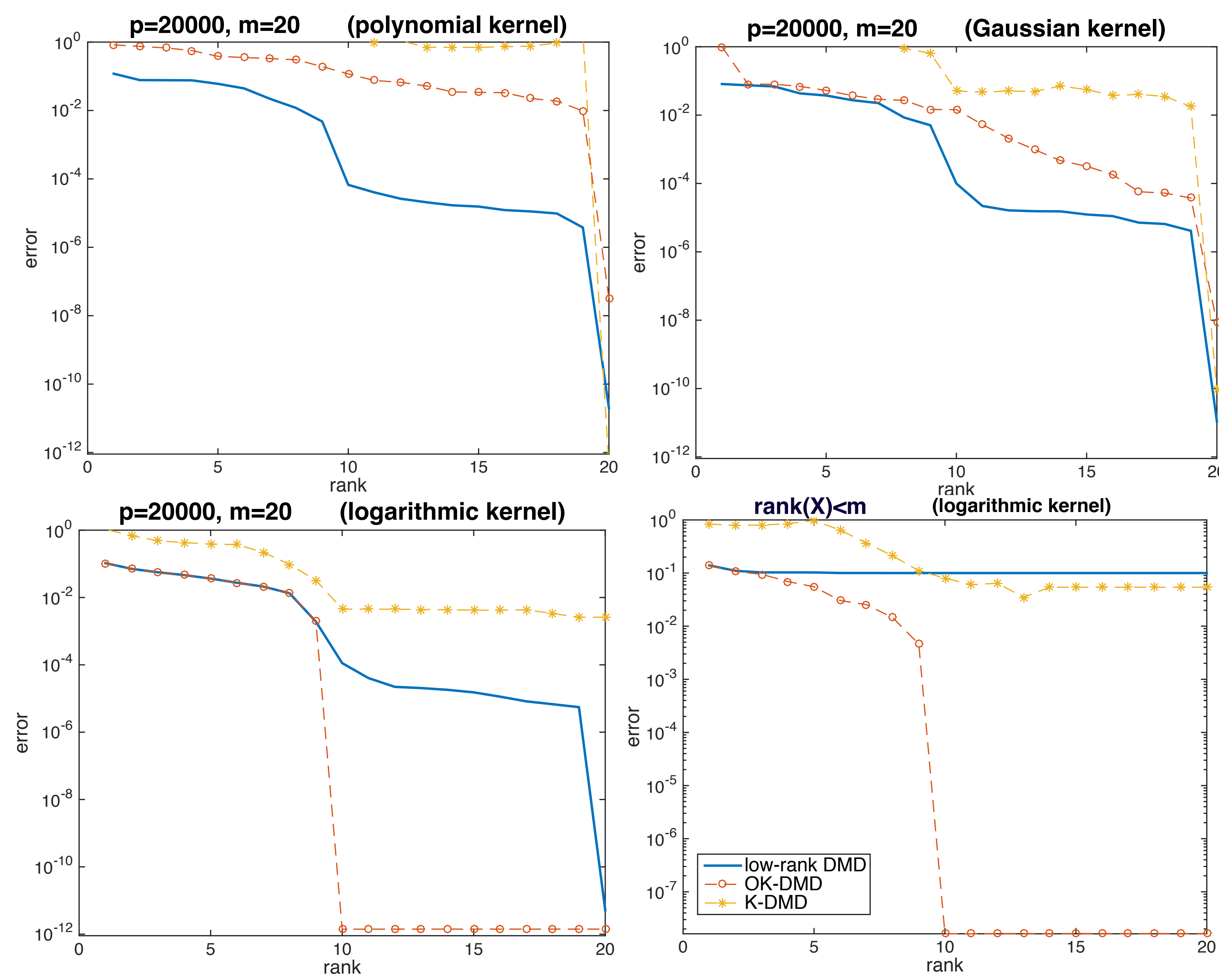

ArXiv reference:

Patrick Héas, Cédric Herzet, "Optimal Kernel-Based Dynamic Mode Decomposition", arXiv:1610.02962, 2017

\section{Inzía ANR}

